\title{
Computing Maximum Association Graph in Microscopic Nucleus Images ${ }^{\star}$
}

\author{
Branislav Stojkovic ${ }^{1}$, Yongding $\mathrm{Zhu}^{1}$, Jinhui $\mathrm{Xu}^{1}$, \\ Andrew Fritz ${ }^{2}$, Michael J. Zeitz ${ }^{3}$, Jaromira Vecerova ${ }^{2}$, and Ronald Berezney ${ }^{2}$ \\ 1 Department of Computer Science and Engineering, \\ State University of New York at Buffalo \\ \{bs65, yzhu3, jinhui\} @buffalo.edu \\ 2 Department of Biological Sciences, State University of New York at Buffalo \\ \{ajfritz, vecerova, berezney\} @buffalo.edu \\ 3 Stanford Medical School, 3801 Miranda Avenue, Palo Alto, CA 94304
}

\begin{abstract}
In this paper, we study the problem of finding organization patterns of chromosomes inside the cell nucleus from microscopic nucleus images. Emerging evidence from cell biology research suggests that global chromosome organization has a vital role in fundamental cell processes related to gene expression and regulation. To understand how chromosome territories are neighboring (or associated) to each other, in this paper we present a novel technique for computing a common association pattern, represented as a Maximum Association Graph (MAG), from the nucleus images of a population of cells. Our approach is based on an interesting integer linear programming formulation of the problem and utilizes inherent observations of the problem to yield optimal solutions. A two-stage technique is also introduced for producing near optimal approximations for large data sets.
\end{abstract}

\section{Introduction}

Chromosome territory refers to a confined region within the cell nucleus where each chromosome is located. Empirical findings suggest that high level spatial organization of chromosome territories affects fundamental molecular biology processes 1223 . In recent years, a significant amount of effort has been focused on studies of its influence on genomic structure and responsibility for specific nuclear functions, such as morphogenesis, transcription and splicing. Investigating association (spatial proximity) among chromosome territories is a necessary component for any study of the higher-order chromatin distribution effect on nuclear functions. However, despite recent developments in microscopy imaging and labeling techniques, accurately detecting such a pattern still remains a challenge.

To investigate this problem, one promising approach is to first represent the high level organization of the chromosome territories in each cell nucleus as a

\footnotetext{
* This research was partially supported by NSF through CAREER award CCF 0546509, ROI grant IIS-0713489 and NIH grant GM-072131.
}

T. Jiang et al. (Eds.): MICCAI 2010, Part II, LNCS 6362, pp. 530-537 2010.

(C) Springer-Verlag Berlin Heidelberg 2010 


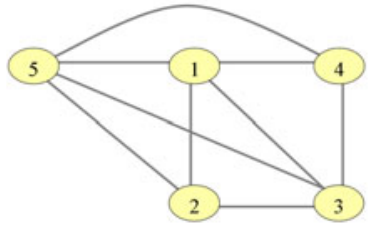

(a)

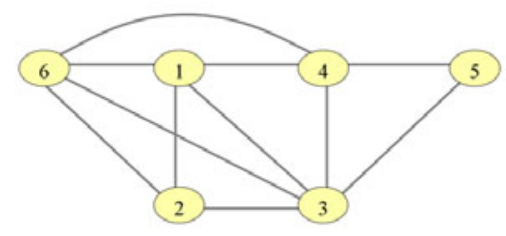

(b)

Fig. 1. When finding a possible alignment from (a) to (b) GMG algorithm will choose the one with cost 4 (relabeling vertex 5 to 6 , adding new vertex 5 and two incident edges). However, in matching where relabeling is disallowed, the only possible alignment is of cost 7 (removing two edges incident to vertex 5 and inserting vertex 6 with four incident edges).

graph, called association graph, and then find the common structural pattern from a set of association graphs corresponding to a population of cells. In each association graph, vertices represent the individual chromosome territories, and edges (between pairs of vertices) indicate the two corresponding chromosome territories are spatially proximate (or associated) to each other. Each vertex has a label which is its chromosome number. Since each chromosome has two homologs (or copies), a label is shared by a pair of vertices.

To find the common structural pattern from a set of association graphs, one way is to use the concept of graph median [4. In the graph median problem, the input is a set of graphs with possible labels associated with nodes. The objective is to compute a new graph, called median graph, so that the distance between the median graph to the input graphs is minimized, where the distance between two graphs is normally defined as their edit distance. For the median graph problem, early results 415] are all of heuristic nature and do not have a bounded running time. Recently, a polynomial time combinatorial algorithm, called Generalized Median Graph(GMG) [], was introduced by Mukherjee et al. Their algorithm uses a generalized distance function which allows vertex labels and edge weights, and produces near optimal solutions.

Despite its obvious advantages, the GMG (or any other median graph) algorithm also has two limitations when used for finding the association patterns from association graphs. First, due to its emphasis on finding the structural similarity between graphs, the GMG tends to match vertices with similar degrees. A possible outcome is that the GMG could match two vertices with different labels. While this is in accordance with the edit distance definition, it gives misleading semantic interpretation in our application to nuclear images, since mismatching has little biological meaning (see Figure 1). Second, the GMG requires graphs with unique predefined labeling. However, in association graphs, each label is shared by a pair of vertices. This is because in nuclear images each chromosome (including its two copies) is uniquely identified by its color, see Fig 2, Thus we are facing the challenge of how to correlate labels of chromosome copies among different cells. Even in the cases when professional expertise is at hand, it is not 

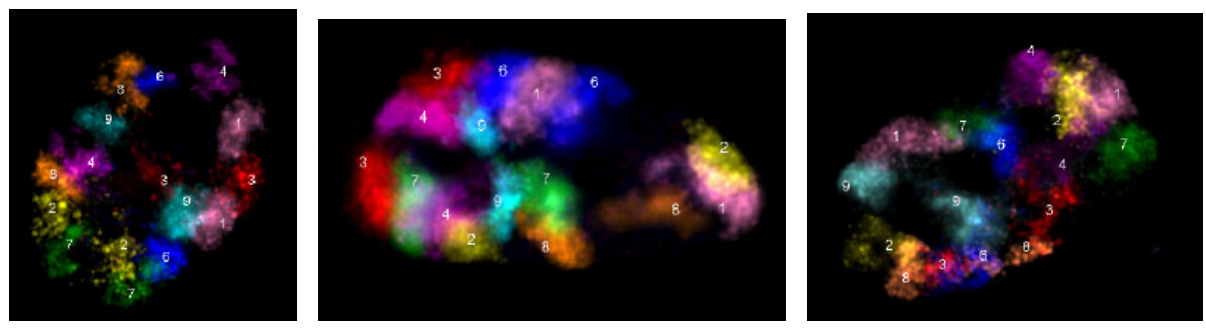

Fig. 2. Three different cell images (from the input set), obtained by superimposing z-stack images, containing eight chromosome colors(labels)

always possible to come up with a reliable mechanism for mapping copies of the same chromosomes between different cells.

To overcome all these difficulties, we propose in this paper a novel technique called Maximum Association Graph. Our approach maximizes the structural similarity between the computed new maximum association graph (MAG) and the set of input association graphs. During the matching process, it explicitly disallows matching of the vertices with different labels, and automatically establishes the correspondence of the same copy of the chromosome over all cells, thus eliminating the possible human errors. Also, it allows the user to specify a frequency threshold for determining the existence of an edge in the association pattern MAG (i.e., an edge in MAG if and only if it appears in a certain percentage of the input graphs). With this threshold, we could obtain the association patterns at different similarity levels.

Our approach is based on an mixed integer linear programming (MILP) formulation, and can be solved optimally using an optimization software package CPLEX for middle sized data sets. For large data sets, we also propose a two-stage approximation technique for obtaining near optimal polynomial-time solution.

\section{Method}

\subsection{Maximal Structural Matching}

Let $L_{V}$ be a finite set of node labels and $\mathbb{I}_{L} \subseteq \mathbb{N}$ be the set of indices. A labeled undirected graph $G$, in which existence of more than one vertex with the same label is permitted, is defined as a triple $G \equiv\left(V, E, f^{v}\right)$ where $V$ is the vertex set, $E$ is the edge set, and $f^{v}: V \longrightarrow\left(L_{V}, \mathbb{I}_{L}\right)$ is a mapping which to every vertex $v$ in $V$ assigns unique pair $(l, k)$, where $l \in L_{V}$ and $k \in \mathbb{I}_{L}$. We call node $v$ a $k$-th instance (copy) of label $l$ in $G$. In this way, each edge $e \in E$ between $v_{1}$ and $v_{2}$ is uniquely identified with 4 -tuple $\left(l_{1}, k_{1}, l_{2}, k_{2}\right)$, where $f\left(v_{1}\right)=\left(l_{1}, k_{1}\right)$ and $f\left(v_{2}\right)=\left(l_{2}, k_{2}\right)$.

Let $\mathbb{G}=\left\{G_{1}, G_{2}, \ldots, G_{n}\right\}$ be a collection of graphs where for every $G_{i}=$ $\left(V_{i}, E_{i}, f_{i}^{v}\right)$, the following conditions hold: $V_{i} \subseteq V$ and $E_{i} \subseteq E$, and there is 
no restriction on the cardinality of nodes in the graphs in G, i.e., $\left|V_{i}\right| \neq\left|V_{j}\right|$, $G_{i}, G_{j} \in \mathbb{G}$.

Structural pattern for $\mathbb{G}$ is obtained as maximal association graph, $\hat{G}=$ $\left(\hat{V}, \hat{E}, f^{v}\right)$, where $\hat{V}=\bigcup_{i} V_{i}$ and $\hat{E} \subseteq \hat{V} \times \hat{V}$. As noted before, finding such a graph, $\hat{G}$, identifies the maximal set of edges between nodes in $\hat{V}$ that are consistent with labeling in all input graphs. This induces label preserving mapping from vertices in every input graph $G_{i}$ to the set of vertices in $\hat{G}$. Which mapping is valid for particular node in some input graph is not known in advance, hence to obtain the optimal solution, we must look for all mapping simultaneously. This condition leads to the choice of mathematical optimization as a natural problem modeling tool. Although technique can be easily extended to deal with more general situation, we restrict our presentation to the case when each label has exactly two copies in every graph.

\subsection{Mixed Integer Linear Programming Formulation}

In our approach, we actively try to match vertices between every input graph and the resulting $\hat{G}$. A matching of an edges follows implicitly. Since, only matching of nodes with the same label is allowed, each node $v$ in $i$-th graph representing $k$-th instance of label $j$, denoted as $v=(j, k), v \in V_{i}$, can be matched to $\hat{k_{1}}$ th or $\hat{k_{2}}$-th instance of $j$-th label in graph $\hat{G}$. These two possible matchings have corresponding binary variables: $\alpha_{i, j, k, \hat{k_{1}}}$ and $\alpha_{i, j, k, \hat{k_{2}}}$. Value of one denotes that particular matching holds in the global solution. Similarly, for each node in $\mathbb{G}$, we introduce corresponding variables for all possible assignments. Thus, the set of constraints guaranteeing that each node in $\mathbb{G}$ has one and only one corresponding node in $\hat{G}$ is given as:

$$
\begin{aligned}
& \sum_{k} \alpha_{i, j, k, \hat{k}}=1, \forall i, j, \hat{k} \\
& \sum_{\hat{k}} \alpha_{i, j, k, \hat{k}}=1, \forall i, j, k
\end{aligned}
$$

For each potential edge in $\hat{E}$ between instance $\hat{k_{1}}$ of vertex labeled $\hat{j_{1}}$ and instance $\hat{i_{2}}$ of vertex labeled $\hat{j_{2}}$ there is a corresponding binary variable $\hat{\gamma}_{\hat{j}_{1}, \hat{j_{2}}, \hat{k_{1}}, \hat{i_{2}}}$, which has the value of 1 if there is an edge between vertices $\left(\hat{j_{1}}, \hat{k_{1}}\right)$ and $\left(\hat{j_{2}}, \hat{k_{2}}\right)$ in $\hat{G}$, and 0 otherwise.

For each induced matching of an edge between instance $k_{1}$ of vertex labeled $j_{1}$ and instance $i_{2}$ of vertex labeled $j_{2}$ in $G_{i}$ to an edge between instances $\hat{k_{1}}$ and $\hat{k_{1}}$ of corresponding vertex labels in $\hat{G}$ there is an associated real variable $\beta_{i, j_{1}, j_{2}, k_{1}, k_{2}, \hat{k_{1}}, \hat{k_{2}}}$.

Formally, we denote an existence of an edge between vertices $\left(j_{1}, k_{1}\right)$ and $\left(j_{2}, k_{2}\right)$ in the input graph $G_{i}$ with $\gamma_{i, j_{1}, j_{2}, k_{1}, k_{2}}$. Now, we can formulate matching constraint for such an edge as:

$$
\beta_{i, j_{1}, j_{2}, k_{1}, k_{2}, \hat{k_{1}}, \hat{k_{2}}}=\alpha_{i, j_{1}, k_{1}, \hat{k_{1}}} * \alpha_{i, j_{2}, k_{2}, \hat{k_{2}}} * \hat{\gamma}_{\hat{j_{1}}, \hat{j_{2}}, \hat{k_{1}}, \hat{k_{2}}} * \gamma_{i, j_{1}, j_{2}, k_{1}, k_{2}}
$$


In other words, an existing edge $\left(j_{1}, j_{2}, k_{1}, k_{2}\right)$ in input graph $G_{i}$ can be matched to an existing edge $\left(\hat{j_{1}}, \hat{j_{2}}, \hat{k_{1}}, \hat{k_{2}}\right)$ in $\hat{G}$ if and only if its belonging vertices $\left(j_{1}, k_{1}\right)$ and $\left(j_{2}, k_{2}\right)$ are matched to $\left(\hat{j_{1}}, \hat{k_{1}}\right)$ and $\left(\hat{j_{2}}, \hat{k_{2}}\right)$ in $\hat{V}$. Constraints given in (2) are in the nonlinear form, however using standard strategy of product term linearization in a constraint we can transform each constraint of this type to a set of inequality constraints.

For an edge in $\hat{G}$ to be present, its matching defined analogue must appear in at least a predefined percentage $P$ of input graphs. This is ensured with the following set of constraint:

$$
\sum_{i} \beta_{i, j_{1}, j_{2}, k_{1}, k_{2}, \hat{k_{1}}, \hat{k_{2}}} \geq \hat{\gamma}_{\hat{j_{1}}, \hat{j_{2}}, \hat{k_{1}}, \hat{k_{2}}} * P *|\mathbb{G}|
$$

where $P$ denotes the specified frequency threshold and $|\mathbb{G}|$ is the cardinality of input set.

Setting the expression for optimization function as the maximization of both number of edges in resulting graph as well as number of possible edge matchings from the set of input graphs we obtain the final mixed integer programming form.

\subsection{Extensions to the Method}

Including Pairwise solutions. In a restricted case we consider a pair of node labels and their belonging copies. Subgraphs obtained by isolating them from all other node instances in each graph from the input set $\mathbb{G}$ are passed as an input to algorithm, eq. (1-3). Solution for this subproblem provides the upper bound on the number of associations between four nodes in $\hat{G}$, two for each label from the chosen pair. In the first phase we solve MILP for each of $\left(\begin{array}{c}\left|L_{V}\right| \\ 2\end{array}\right)$ pairs in order to obtain the upper bound for each of $\hat{\gamma}_{\hat{j_{1}}, \hat{j_{2}}, \hat{k_{1}}, \hat{k_{2}}}, \hat{G}$ edge indicator variable. In the second phase we find the MILP solution, eq. (1-3), for original input set, but this time, adding set of upper bound constraints obtained in the first phase. Introducing new constraints is meaningful since it reduces feasible space and decreases overall running time without affecting the quality of the solution.

Two-stage algorithm. Mixed integer linear programming proposed above, eq. (1-3), can be solved optimally applying standard techniques of branch and cut available in commercial or free solvers. However, when dealing with large graphs or with increasing the size of input sets running time may considerably increase. Therefore we develop a modification for trading a little of accuracy for great reduction in the running time. We divide input sets in subgroups, and then solve problem on each of them optimally. We merge subgroup solutions by solving the MILP, eq. (1-3), again but this time on subgroup solutions given as input. We assign a proper weight to every edge denoting the number of graphs in which it appears. Exhaustive enumeration analysis suggests that a solution obtained in this way is close to the one obtained by solving MILP without partitioning. Over $95 \%$ of edges were preserved in approximative solution comparing to the optimal one. 


\section{Experimental Results}

To test the validity of the method we calculated a maximum association graph on the input set of 15 randomly generated graphs. Graphs were created in the form of adjacency matrices mimicking the sparsity property of graphs obtained from cell nucleus images by having roughly the same number of edges. Adjacency matrices had at most 16 vertices with each vertex label appearing at most twice, which correlates with our real data graph size. A random graph $A$ and then its complement $\tilde{A}$ were generated. We populated the first set with $P * 15$ number of permuted versions of graph $A$, and the second set with $(100 \%-P) * 15$ number of permuted versions of graph $\tilde{A}$. Merging two sets together we obtained the input set. Using CPLEX as MILP solving tool, we were able to get the optimal solution i.e. calculated maximum association graph was identical to the generated input graph $A$ in all test cases.

Further, the validation process considered graphs with added noise. We designed a procedure similar to those proposed in [7/8]. Following the steps from the previous paragraph a random graph $A$ was created. We used graph $A$ as the basis for populating the input set: For each new member of input set a copy of $A, A^{\prime}$, was made and randomly permuted with label preserving permutation matrix. To introduce the noise, removal of each edge in $A^{\prime}$ was considered with the probability denoted as noise parameter, $E$. In this way we ensured that all generated graphs were proper subgraphs of $A$ having the expected number of associations equal to the value of noise parameter, $E$. Maximum association graph was compared with each graph in the input set individually. Cardinality of maximum matching was calculated (using permutation matrices) and normalized by the size of maximum possible matching for the whole input set (obtained by matching with the base graph $A$ ). Average values for two methods are shown in Table 1. They correlate (are slightly above or below) to the values of expected maximum number of associations that are allowed by introduced random perturbations. Also, we notice the agreement between the results of two proposed methods.

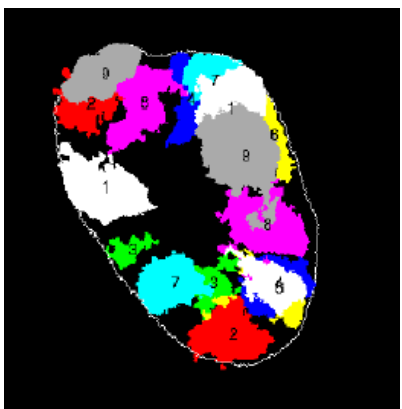

(a)

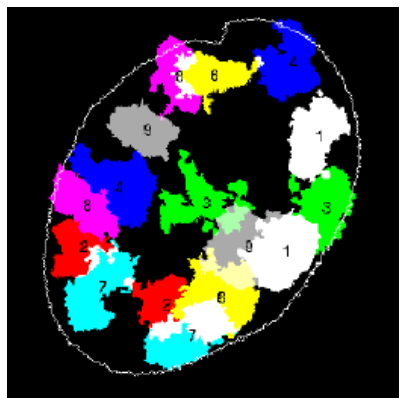

(b)

Fig. 3. 2D segmented sections of chromosome territory in cell nucleus images 


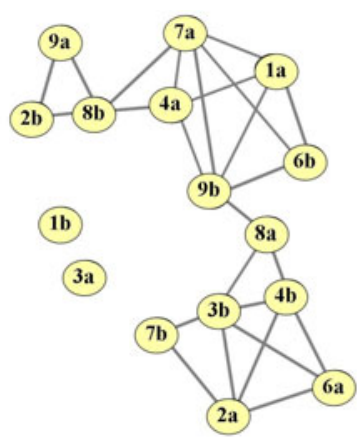

(a)

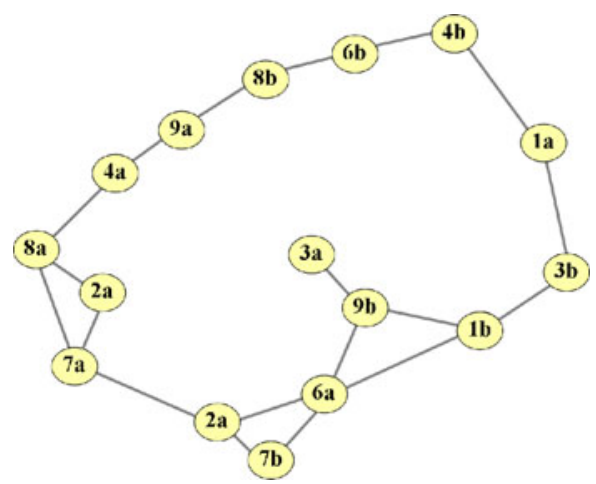

(b)

Fig. 4. Derived graphical representation of chromosome territory in cell nucleus

For qualitative evaluation of the proposed method we discuss experiments related to our motivating biological image analysis application. A previously developed procedure 9] using so called FISH technique was used for labeling 8 pairs of the larger chromosomes (numbers $1,2,3,4,6,7,8,9$ ) from the human lung fibroblast cell line WI38. The 3D microscopy z-stack image acquisition process was repeated for a total number of 45 cells, see Fig 2 After the registration and segmentation processes were performed, masks for individual chromosome pairs were created for each raw image, see Fig 3 . Nearest border to border distances were calculated for every combination of chromosome pairs. To obtain the individual graph representation for each cell, pairwise associations were derived using a threshold value for distance of 4 pixels, which corresponds to $0.28 \mu \mathrm{m}$, see Fig 4. In the graph, each individual chromosome is represented as a labeled vertex. We distinguish between two instances of the same label by denoting them as copy a and copy $b$, respectively. Spatial proximity (adjacency) between chromosomes is expressed as an edge between vertices.

We report on the following observations. MAG results for different values of frequency threshold $(100 \%-40 \%)$ indicate consistency among the structural patterns. For example, the majority of the edges that appear at $P=60 \%$ also

Table 1. Average matching similarity with a graph from the input set

\begin{tabular}{|c|c|c|c|}
\cline { 2 - 4 } \multicolumn{1}{c|}{} & \multicolumn{3}{c|}{ Percentage of Matching } \\
\hline Noise parameter (E) & $15 \%$ & $30 \%$ & $40 \%$ \\
\hline MAG & 0.84 & 0.72 & 0.62 \\
\hline Two-Stage Method & 0.83 & 0.69 & 0.60 \\
\hline
\end{tabular}

Table 2. Average Sorensen's index value with a graph from the input set

\begin{tabular}{|c|c|c|c|c|}
\hline Algorithm & MAG 65\% & MAG 55\% & MAG 50\% & GMG \\
\hline Sorensen Index & 0.28 & 0.37 & 0.46 & .40 \\
\hline
\end{tabular}


appear at lower values for the threshold and we can almost perfectly align output graph for higher $P$ as a sub-graph of computed maximum associated graph for lower $P$. Moreover, the model captures the essential association information from the constituent graphs. This is reflected in calculated Sorensen index values which show increased similarity with the decrease of frequency parameter $P$. Table 2 contains obtained values and provides comparison with the GMG method 96].

\section{Conclusions}

In this paper we used the specific form of mathematical optimization approach for computing the maximum association graph in context of a real world vision problems in biological image analysis. This formulation can be easily extended to handle the more general case, where arbitrary number of instances of vertices with the same label are present. This is relevant for the study of cancer cells where more than two copies of certain chromosomes are typically present within the cell nucleus. In the future, we plan to examine chromosome territories associations for larger number of pairs and further study the structural differences among different chromosome territory subgroups using the techniques described in this contribution. We believe that the idea of maximum association graph will find general applicability in learning patterns from graph representations, in particular constructing comprehensive three-dimensional arrangement of all 23 pairs of chromosome in a diploid somatic cell nucleus.

\section{References}

1. Berezney, R.: Regulating the mammalian genome: the role of nuclear architecture. Advances in Enzyme Regulation 42, 39-52 (2002)

2. Cremer, T., Cremer, C.: Chromosome territories, nuclear architecture and gene regulation in mamalian cells. Nature Reviews Genetics 2, 292-301 (2001)

3. Stein, G.S., Zaidi, S.K., Braastad, C.D., Montecino, M., van Wijnen, A.J., Choi, J.Y., Stein, J.L., Lian, J.B., Javed, A.: Functional architecture of the nucleus: Organizing the regulatory machinery for gene expression, replication and repair. Trends Cell Biol. 13, 584-592 (2003)

4. Jiang, X., Münger, A., Bunke, H.: On median graphs: Properties, algorithms, and applications. IEEE Trans. Pattern Anal. Mach. Intell. 23(10), 1144-1151 (2001)

5. Hlaoui, A., Wang, S.: Median graph computation for graph clustering. Soft. Comput. 10(1), 47-53 (2006)

6. Mukherjee, L., Singh, V., Peng, J., Xu, J., Zeitz, M.J., Berezney, R.: Generalized median graphs: Theory and applications. In: ICCV, pp. 1-8 (2007)

7. Ullmann, J.R.: An algorithm for subgraph isomorphism. J. ACM 23(1), 31-42 (1976)

8. Foggia, P., Sansone, C., Vento, M.: A database of graphs for isomorphism and subgraph isomorphism benchmarking. In: CoRR, pp. 176-187 (2001)

9. Zeitz, M.J., Mukherjee, L., Bhattacharya, S., Xu, J., Berezney, R.: A probabilistic model for the arrangement of a subset of human chromosome territories in wi38 human fibroblasts. Journal of Cellular Physiology 221(1), 120-129 (2009) 